\title{
Matrix Gla Protein, Plaque Stability, and Cardiovascular Events in Patients with Severe Atherosclerotic Disease
}

\author{
Sabine R. Zwakenberg ${ }^{a} \quad$ Yvonne T. van der Schouw $^{\mathrm{a}}$ Cees Vermeer ${ }^{\mathrm{b}}$ \\ Gerard Pasterkamp ${ }^{c}$ Hester M. den Ruijter ${ }^{c}$ Joline W.J. Beulens ${ }^{a, d}$ \\ a Julius Center for Health Sciences and Primary Care, University Medical Center Utrecht, Utrecht, The Netherlands; \\ ${ }^{b} R$ \& D Group VitaK, Maastricht University, Maastricht, The Netherlands; ' Laboratory of Experimental Cardiology, \\ University Medical Center Utrecht, Utrecht, The Netherlands; ${ }^{d}$ Department of Epidemiology and Biostatistics, \\ Amsterdam Public Health Research Institute, VU University Medical Center, Amsterdam, The Netherlands
}

\section{Keywords}

Matrix Gla protein · Plaque stability · dp-ucMGP · t-ucMGP · Cardiovascular events

\section{Abstract}

Objective: This study aims to investigate whether plasma matrix Gla protein (MGP) species, desphospho-uncarboxylated (dp-uc) MGP, and total uncarboxylated (t-uc) MGP are associated with plaque levels of uncarboxylated (uc) MGP, markers of plaque stability, and cardiovascular disease (CVD) risk. Methods: From the Athero-Express biobank, we selected carotid plaque samples of 100 patients who underwent carotid endarterectomy. The level of agreement between plasma MGP species and plaque ucMGP levels was assessed using weighted kappa ( $\mathrm{K}$ ). We analyzed histological characteristics of plaque composition (plaque hemorrhage, lipid and calcification content). Logistic regression analyses were used to assess the association between plasma MGP and plaque characteristics. Furthermore, CVD endpoints $(n=20)$ were collected over a mean follow-up of 2.6 years. Results: Weighted $\mathrm{k}$ statistics of plasma dp-ucMGP and t-ucMGP and plaque ucMGP were $0.10(95 \% \mathrm{Cl}-0.31$ to 0.52$)$ and 0.14
( $95 \% \mathrm{Cl}-0.20$ to 0.48$)$. Higher dp-ucMGP levels tended to be associated with less plaque hemorrhage $\left(\mathrm{OR}_{\text {per }} 500 \mathrm{nM} 0.96\right.$; $95 \% \mathrm{Cl} 0.92-1.00)$. No association was found for lipid and calcification content. Cox proportional hazards models showed no association between dp-ucMGP $\left(\mathrm{HR}_{\text {per }} 200 \mathrm{pM} 0.92\right.$; $95 \% \mathrm{Cl} 0.75-1.11)$ and an inverse association between t-ucMGP ( $\left.\mathrm{HR}_{\text {per } 500 \mathrm{nM}} 0.79 ; 95 \% \mathrm{Cl} 0.62-0.99\right)$ and cardiovascular events. Conclusions: Plasma dp-ucMGP and t-ucMGP concentrations do not reflect plaque ucMGP levels. Elevated dpucMGP levels may be associated with less plaque hemorrhage, suggestive of more stable plaques. T-ucMGP was not related with markers of plaque stability; however, elevated plasma t-ucMGP levels were associated with a reduced CVD risk.

(c) 2018 The Author(s)

Published by S. Karger AG, Basel

\section{Introduction}

Vascular calcification is associated with an increased cardiovascular disease (CVD) risk, and matrix Gla protein (MGP) is an inhibitor of vascular calcification [1]. Proposed mechanisms for inhibition of vascular calcifica-

\begin{tabular}{ll}
\hline KARGER & $\begin{array}{l}\text { @ } 2018 \text { The Author(s) } \\
\text { Published by S. Karger AG, Basel }\end{array}$ \\
E-Mail karger@karger.com & $\begin{array}{l}\text { This article is licensed under the Creative Commons Attribution- } \\
\text { NonCommercial-NoDerivatives 4.0 International License (CC BY- } \\
\text { NC-ND) (http://www.karger.com/Services/OpenAccessLicense). } \\
\text { Usage and distribution for commercial purposes as well as any dis- } \\
\text { tribution of modified material requires written permission. }\end{array}$
\end{tabular}


tion all require vitamin $\mathrm{K}$-dependent carboxylation of MGP [2].

MGP exists in various species, differing in their phosphorylation and/or carboxylation state. High desphospho-uncarboxylated (dp-uc) MGP, representing a poor vitamin $\mathrm{K}$ status, is associated with an increased CVD risk $[3,4]$. Total uncarboxylated (t-uc) MGP includes dp-ucMGP but mainly consists of phosphorylated uncarboxylated (uc) MGP [5]. Circulating t-ucMGP has been proposed as a biomarker for prevalent vascular calcification $[3,6]$; however, the association between t-ucMGP and $\mathrm{CVD}$ risk showed inconsistent results ranging from no to an inverse association with cardiovascular events $[5,7]$. Vitamin $\mathrm{K}$ antagonist treatment resulted in high dp-ucMGP levels and accelerated plaque calcification [4] and shifted atherosclerotic plaque to vulnerable plaques in mice [8]. No studies have been performed investigating plasma MGP levels and plaque stability in humans.

The aim of this study was to assess whether plasma dpucMGP and t-ucMGP levels are associated with ucMGP levels in plaque, markers of plaque stability, and cardiovascular events in a very high-risk population of patients with occlusive atherosclerotic disease in the carotid artery.

\section{Materials and Methods}

\section{Study Population}

Athero-Express is an ongoing biobank study of patients undergoing carotid and femoral endarterectomy in two Dutch centers from 2002 onwards. Details of the study design have been described previously [9]. Blood samples are taken before surgery and were stored at $-80^{\circ} \mathrm{C}$. Patients gave informed consent prior to study inclusion and this study was approved by the local medical ethics committee. All patients underwent a clinical follow-up 1 year after surgical intervention and completed postal questionnaires 1,2 , and 3 years after the operation.

This study included 100 patients who underwent carotid endarterectomy for stroke, transient ischemic attack, and amurosis fugax or asymptomatic patients between 2002 and 2006. A patient group was selected based on the estimated glomerular filtration rate (eGFR) and calcification level (assessed with histological assessment); 25 patients with eGFR between 30 and $60 \mathrm{~mL} /$ $\mathrm{min} / 1.73 \mathrm{~m}^{2}, 25$ patients with eGFR levels above $60 \mathrm{~mL} / \mathrm{min} /$ $1.73 \mathrm{~m}^{2}, 25$ patients with no plaque calcification, and 25 patients with heavy calcification staining were selected for this study. Selection was based on eGFR and calcification levels since these factors highly affect plasma MGP levels and thus ensure sufficient variation in MGP levels.

Patients with missing data on plasma MGP levels $(n=2)$, plaque ucMGP levels $(n=9)$, or cardiovascular events occurrence $(n=3)$ were excluded, leaving 90 patients for the comparison between plasma MGP levels and plaque ucMGP levels, 98 patients for plasma MGP and plaque stability markers, and 95 patients for analyses with plasma MGP levels and cardiovascular events.

\section{Plaque Stability}

Atherosclerotic plaques were harvested during carotid endarterectomy according to a standardized protocol. The segment with the greatest plaque burden was considered the culprit lesion. A detailed description of the histological assessment has been published previously [9]. Plaque hemorrhage was defined as hemorrhage within the tissue of the plaque and scored as absent or present. The lipid core size was expressed as the percentage of total plaque area and scored as $<40$ and $\geq 40 \%$, and calcification was semi-quantitatively scored as no/minor or moderate/heavy staining.

\section{Matrix Gla Protein}

ucMGP in plaque was visualized by immunohistochemistry using a monoclonal ucMGP antibody, and the intensity of staining was scored by 2 independent coworkers as no/minor, moderate, or heavy staining. Conflicting scores were resolved in consensus with a third person. Plasma dp-ucMGP measurements were performed with a sandwich dual-antibody ELISA and plasma t-ucMGP levels were examined with a competitive mono-antibody ELISA, as described previously [10].

\section{Statistical Analyses}

Baseline characteristics were summarized as percentages or medians (IQR), since the variables were not normally distributed. Dp-ucMGP and t-ucMGP levels were divided in tertiles. Three types of analyses were performed; first, weighted kappa $(\kappa)$ statistics were calculated to assess agreement between tertiles of plasma dp-ucMGP and t-ucMGP levels and plaque ucMGP levels; second, we assessed the association between dp-ucMGP and t-ucMGP and markers of plaque stability using logistic regression analyses adjusted for age and eGFR; finally, associations of dp-ucMGP and t-ucMGP with cardiovascular events (fatal and nonfatal) were analyzed with Cox proportional hazard regression models. Based on the literature, we investigated whether age, gender, current smoking (yes/no), eGFR (using the MDRD formula), and degree of contralateral stenosis (0-50/50-70/70$99 / 100 \%)$ were confounders in this association. Only age and eGFR were associated with dp-ucMGP and t-ucMGP and included as confounders. Gender, smoking status, and degree of stenosis were included in a sensitivity analysis. All analyses were performed in $\mathrm{R}$ version 3.2.2. $p<0.05$ was considered statistically significant.

\section{Results}

The study population had a mean age of 70 years, and $57 \%$ was male, with a mean BMI of 26 (Table 1). Participants with higher dp-ucMGP levels were older, less often current smokers and had lower eGFR levels, while patients with higher t-ucMGP levels had higher eGFR levels.

There was no association between dp-ucMGP (weighted $\kappa=0.10 ; 95 \% \mathrm{CI}-0.31$ to 0.52$)$ or t-ucMGP $(0.14 ; 95 \%$ CI -0.20 to 0.48 ) concentrations and plaque ucMGP levels. The levels of plasma dp-ucMGP and t-ucMGP by plaque ucMGP levels are shown in Figure 1. 
Table 1. Baseline characteristics by tertiles of dp-ucMGP and t-ucMGP

\begin{tabular}{|c|c|c|c|c|c|c|}
\hline & \multicolumn{3}{|l|}{ dp-ucMGP } & \multicolumn{3}{|l|}{ t-ucMGP } \\
\hline & $\begin{array}{l}\text { tertile } 1 \\
(95-364 \mathrm{pM}, n=33)\end{array}$ & $\begin{array}{l}\text { tertile } 2 \\
(365-573 \mathrm{pM}, n=32)\end{array}$ & $\begin{array}{l}\text { tertile } 3 \\
(576-2,800 \mathrm{pM}, n=33)\end{array}$ & $\begin{array}{l}\text { tertile } 1 \\
(956-1,504 \mathrm{nM}, n=33)\end{array}$ & $\begin{array}{l}\text { tertile } 2 \\
(2,496-3,575 \mathrm{nM}, n=32)\end{array}$ & $\begin{array}{l}\text { tertile } 3 \\
(3,593-7,653 \mathrm{nM}, n=33)\end{array}$ \\
\hline Age, years & $64.0(60.0-72.0)$ & $70.0(65.8-76.0)$ & $73.0(68.0-79.0)$ & $72.0(64.0-77.0)$ & $74.5(62.8-79.0)$ & $68.0(61.0-71.0)$ \\
\hline Males & $19(57.6)$ & $23(71.9)$ & $15(45.5)$ & $19(57.6)$ & $17(53.1)$ & $21(63.6)$ \\
\hline BMI & $24.6(23.6-27.6)$ & $26.6(23.4-29.4)$ & $26.0(22.4-28.3)$ & $26.6(23.3-28.3)$ & $25.2(23.4-27.8)$ & $25.7(23.5-28.4)$ \\
\hline Current smokers & $14(42.4)$ & $10(31.2)$ & $10(30.3)$ & $11(33.3)$ & $9(28.1)$ & $14(42.4)$ \\
\hline Diabetes & $8(24.2)$ & $10(31.2)$ & $11(33.3)$ & $8(24.2)$ & $6(18.8)$ & $15(45.5)$ \\
\hline Hypertension & $23(69.7)$ & $23(71.9)$ & $30(90.9)$ & $25(75.8)$ & $28(87.5)$ & $23(69.7)$ \\
\hline Contralateral stenosis & $9(27.3)$ & $8(25.0)$ & $8(24.2)$ & $10(30.3)$ & $9(28.1)$ & $7(21.2)$ \\
\hline \multicolumn{7}{|l|}{ Laboratory results } \\
\hline $\mathrm{eGFR}, \mathrm{mL} / \mathrm{min} / 1.73 \mathrm{~m}^{2}$ & $77(65-90)$ & $67(55-78)$ & $55(50-65)$ & $64(53-82)$ & $65(55-73)$ & $78(52-85)$ \\
\hline Total cholesterol, mg/dL & $4.6 \pm 0.9$ & $4.6 \pm 1.1$ & $4.2 \pm 1.2$ & $4.4 \pm 1.2$ & $4.4 \pm 1.0$ & $4.5 \pm 1.1$ \\
\hline $\mathrm{HDL}, \mathrm{mg} / \mathrm{dL}$ & $1.2(1.0-1.4)$ & $1.1(1.0-1.3)$ & $0.9(0.7-1.3)$ & $1.2(0.9-1.3)$ & $1.0(0.9-1.4)$ & $1.1(1.0-1.3)$ \\
\hline $\mathrm{LDL}, \mathrm{mg} / \mathrm{dL}$ & $2.7(2.1-3.4)$ & $3.1(2.2-3.4)$ & $2.5(1.6-3.2)$ & $2.7(2.0-3.6)$ & $2.6(2.1-3.3)$ & $2.6(2.0-3.3)$ \\
\hline DpucMGP, pM & $296(199-341)$ & $459(426-531)$ & $833(661-1,180)$ & $433(330-646)$ & $493(350-818)$ & $445(353-576)$ \\
\hline t-ucMGP, nM & $3,021(2,225-4,455)$ & $3,315(2,569-4,060)$ & $2,783(2,144-3,612)$ & $2,060(1,710-2,225)$ & $3,073(2,780-3,279)$ & $4,466(4,117-5,366)$ \\
\hline \multicolumn{7}{|l|}{ Plaque characteristics } \\
\hline Calcified plaque & $16(48.5)$ & $16(50.0)$ & $19(57.6)$ & $20(60.6)$ & $16(50.0)$ & $15(45.5)$ \\
\hline Fat content $<40 \%$ & $23(69.7)$ & $21(65.6)$ & $27(81.8)$ & $23(69.7)$ & $22(68.8)$ & $26(78.8)$ \\
\hline Plaque hemorrhage & $29(87.9)$ & $23(71.9)$ & $14(42.4)$ & $22(66.7)$ & $24(75.0)$ & $20(60.6)$ \\
\hline \multicolumn{7}{|l|}{ Plaque ucMGP } \\
\hline Moderate/heavy staining & $22(66.7)$ & $29(90.6)$ & $17(51.5)$ & $21(63.6)$ & $24(75.0)$ & $23(69.7)$ \\
\hline
\end{tabular}

Values are presented as medians (IQR), means \pm SD, or numbers (\%). HDL, high-density lipoprotein; LDL, low-density lipoprotein.

Table 2. Results of logistic regression analyses for plasma MGP and plaque characteristics and results of the Cox proportional hazards models for plasma MGP and cardiovascular events $(n=20)$

\begin{tabular}{lllll}
\hline & Calcified plaque & & & \\
& & Fat content $<40 \%{ }^{\mathrm{a}}$ & Plaque hemorrhage $^{\mathrm{a}}$ & Cardiovascular events $^{\mathrm{b}}$ \\
\hline dp-ucMGP & $0.99(0.94-1.04)$ & $0.98(0.94-1.02)$ & $0.96(0.92-1.00)$ & $0.92(0.75-1.11)$ \\
t-ucMGP & $0.97(0.94-1.01)$ & $0.99(0.95-1.03)$ & $0.98(0.94-1.02)$ & $0.79(0.62-0.99)$ \\
\hline
\end{tabular}

${ }^{a}$ Values are presented as OR (95\% CI). ${ }^{b}$ Values are presented as HR (95\% CI). OR/HR represent every 200 pM increment in dpucMGP and every $500 \mathrm{nM}$ increment in t-ucMGP. All models are adjusted for age and eGFR.

Fig. 1. dp-ucMGP and t-ucMGP presented by the different categories of plaque ucMGP.

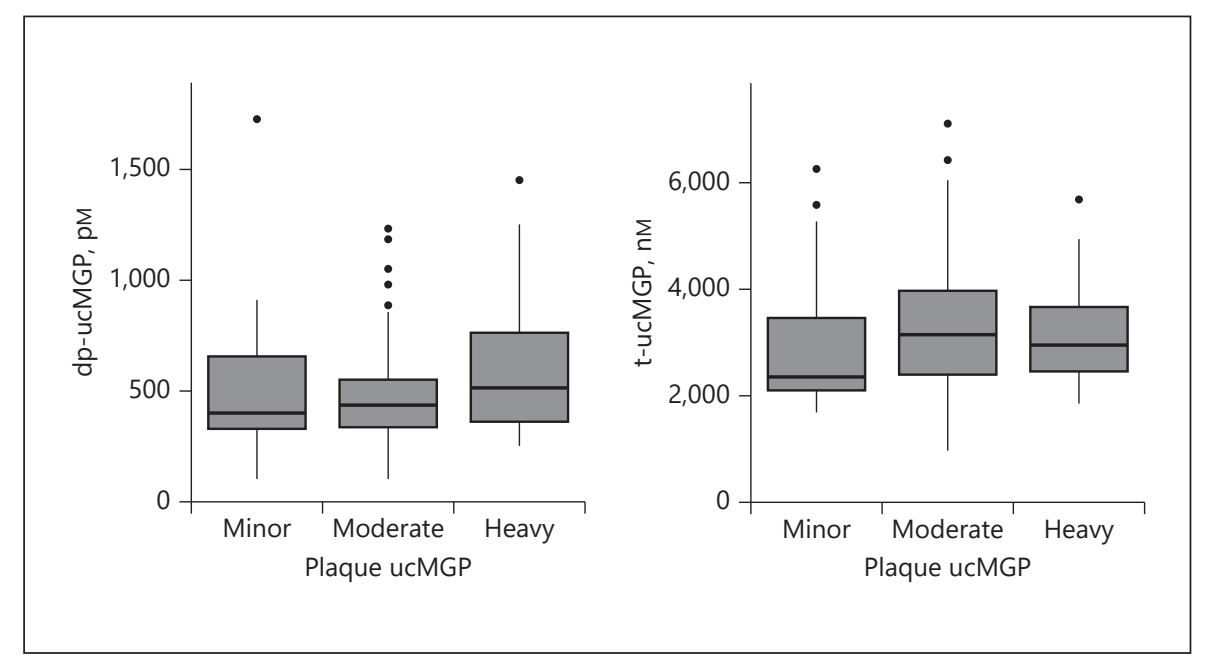


Higher plasma dp-ucMGP levels were suggested to be associated with less plaque hemorrhage (OR 0.96; 95\% CI 0.92-1.00) (Table 2). No associations were observed between dp-ucMGP levels and fat content and plaque calcification. Plasma t-ucMGP was not associated with plaque characteristics.

During 2.6 years of follow-up, 20 cardiovascular events occurred, of which 17 participants deceased. Dp-ucMGP (per $200 \mathrm{pM}$ ) was not associated with cardiovascular events (HR 0.92; 95\% CI 0.75-1.11) adjusted for age and eGFR (Table 2). Each $500 \mathrm{~nm}$ higher t-ucMGP level was associated with a reduced CVD risk (HR 0.79; 95\% CI 0.62-0.99) adjusted for age and eGFR. Including gender, smoking status, and degree of stenosis did not substantially change the results (dp-ucMGP: 0.88 [95\% CI $0.72-$ 1.08], t-ucMGP: 0.79 [95\% CI 0.62-0.99]).

\section{Discussion}

This is a first study comparing plasma MGP levels with plaque MGP levels and plaque stability, but the study is exploratory in nature because of the small sample size and semi-quantitative measures. The sample size was also too small to investigate the association between plaque ucMGP and CVD events. We hypothesized that elevated dp-ucMGP was linked to instable plaques and an increased CVD risk $[3,4,8]$. In our study, higher plasma dp-ucMGP levels tended to be associated with less plaque hemorrhage. This inverse association seems counterintuitive at first. However, active MGP (low dp-ucMGP levels) is an inhibitor of calcification [1]. Calcification rich plaques may cause more plaque stability; therefore, low dp-ucMGP levels may represent stable plaques [11]. Nevertheless, no association was found between dp-ucMGP and calcification levels. The association between dp-ucMGP and calcification might be different in patients with severe atherosclerotic disease or this could be due to a lack of power.

Previous studies proposed t-ucMGP as a marker of prevalent vascular calcification $[3,6]$ instead of an active player in vascular calcification. However, our study did not find an association between t-ucMGP and different plaque characteristics. To date, the literature is still inconclusive regarding the association between $\mathrm{t}$-ucMGP and CVD risk $[3,6]$. It is suggested that t-ucMGP may act differently in persons with or without calcification [5]. Calcified vessels may hamper MGP phosphorylation [12]. Since t-ucMGP mainly consists of phosphorylated ucMGP levels, this could result in lower circulating t-uc-
MGP levels [12]. Future research, with an enhanced sample size, is necessary to give more insight into the association between $\mathrm{t}$-ucMGP and CVD risk.

In conclusion, plasma dp-ucMGP and t-ucMGP concentrations did not reflect plaque ucMGP levels. Elevated dp-ucMGP levels may be associated with less plaque hemorrhage, suggestive of more stable plaques. T-ucMGP was not linked to plaque stability; nevertheless, high plasma t-ucMGP levels were associated with a reduced CVD risk.

\section{Statement of Ethics}

Subjects gave their written informed consent.

\section{Disclosure Statement}

The authors have no ethical conflicts to disclose.

\section{Funding Sources}

S.R. Zwakenberg and J.W.J. Beulens are supported by a Senior Dr. Dekker grant (2013T120) from the Dutch Heart Foundation.

\section{Author Contributions}

S.R. Zwakenberg, Y.T. van der Schouw, and J.W.J. Beulens performed the analyses and wrote this manuscript. C. Vermeer, G. Pasterkamp, and H.M. den Ruijter collected the data and reviewed this paper.

References

1 Proudfoot D, Skepper JN, Shanahan CM, Weissberg PL: Calcification of human vascular cells in vitro is correlated with high levels of matrix Gla protein and low levels of osteopontin expression. Arterioscler Thromb Vasc Biol 1998;18:379-388.

2 Reutelingsperger C, Schurgers L: Coronary Artery Calcification: A Janus-Faced Biomarker? JACC Cardiovasc Imaging 2018;11:13241326.

3 Dalmeijer GW, van der Schouw YT, Vermeer C, Magdeleyns EJ, Schurgers LJ, Beulens JWJ: Circulating matrix Gla protein is associated with coronary artery calcification and vitamin $\mathrm{K}$ status in healthy women. J Nutr Biochem 2013;24:624-628. 
4 Rennenberg RJ, van Varik BJ, Schurgers LJ, Hamulyak K, Ten Cate H, Leiner T, Vermeer C, de Leeuw PW, Kroon A: Chronic coumarin treatment is associated with increased extracoronary arterial calcification in humans. Blood 2010;115:5121-5123.

5 Dalmeijer GW, Van Der Schouw YT, Magdeleyns EJ, Vermeer C, Verschuren WMM, Boer JM, Beulens JWJ: Matrix Gla protein species and risk of cardiovascular events in type 2 diabetic patients. Diabetes Care 2013; 36:3766-3771.

6 Cranenburg ECM, Vermeer C, Koos R, Boumans ML, Hackeng TM, Bouwman FG, Kwaijtaal M, Brandenburg VM, Ketteler M, Schurgers LJ: The circulating inactive form of matrix Gla protein (ucMGP) as a biomarker for cardiovascular calcification. J Vasc Res 2008;45:427-436.

7 Mayer O, Seidlerová J, Vaněk J, Karnosová P, Bruthans J, Filipovský J, Wohlfahrt P, Cífková R, Windrichová J, Knapen MHJ, Drummen
NEA, Vermeer C: The abnormal status of uncarboxylated matrix Gla protein species represents an additional mortality risk in heart failure patients with vascular disease. Int J Cardiol 2016;203:916-922.

8 Schurgers LJ, Joosen IA, Laufer EM, Chatrou MLL, Herfs M, Winkens MHM, Westenfeld R, Veulemans V, Krueger T, Shanahan CM, Jahnen-Dechent W, Biessen E, Narula J, Vermeer C, Hofstra L, Reutelingsperger CP: Vitamin $\mathrm{K}$-antagonists accelerate atherosclerotic calcification and induce a vulnerable plaque phenotype. PLoS One 2012;7:1-14.

9 Verhoeven BA, Velema E, Schoneveld AH, de Vries JP, de Bruin P, Seldenrijk C, de Kleijn DPV, Busser E, van der Graaf Y, Moll F, Pasterkamp G: Athero-Express: differential atherosclerotic plaque expression of mRNA and protein in relation to cardiovascular events and patient characteristics: rationale and design. Eur J Epidemiol 2004;19:11271233.
10 Cranenburg ECM, Koos R, Schurgers LJ, Magdeleyns EJ, Schoonbrood THM, Landewé RB, Brandenburg VM, Bekers O, Vermeer C: Characterisation and potential diagnostic value of circulating matrix Gla protein (MGP) species. Thromb Haemost 2010;104:811-822.

11 Liu YP, Gu YM, Thijs L, Knapen MHJ, Salvi E, Citterio L, Petit T, Delli Carpini S, Zhang Z, Jacobs L, Jin Y, Barlassina C, Manunta P, Kuznetsova T, Verhamme P, Struijker-Boudier HA, Cusi D, Vermeer C, Staessen JA: Inactive matrix Gla protein is causally related to adverse health outcomes: a Mendelian randomization study in a Flemish population. Hypertension 2015;65:463-470.

12 Cranenburg ECM, Brandenburg VM, Vermeer C, Stenger M, Mühlenbruch G, Mahnken AH, Gladziwa U, Ketteler M, Schurgers LJ: Uncarboxylated matrix Gla protein (ucMGP) is associated with coronary artery calcification in haemodialysis patients. Thromb Haemost 2009;101:359-366. 